\title{
Colgajo dorsal ancho por abordaje único vertical en el síndrome de Poland. Caso clínico
}

\section{Latissimus dorsi vertical single-approach flap in Poland syndrome. Clinical case. \\ Retalho Latíssimo do Dorso por abordagem único vertical na síndrome de Poland. Caso clínico}

\author{
Maximiliano Juri¹, Virginia Giachero²
}

\section{RESUMEN}

El sindrome de Poland constituye una malformación que asocia diversos grados de anomalia toráxica y a nivel del miembro superior. con una incidencia de 1 cada 30000 nacidos vivos.

Esta patología genera ausencia de la glándula mamaria y del músculo pectoral mayor como malformación más frecuente. Lo cual produce una asimetría a nivel de tórax con perdida de la armonía, la cual lleva a una alteración tanto física como psicológica a nivel de la paciente. Por esto es necesario resolver esta patologia con la menor morbilidad posible.

El planteo de generar la menor morbilidad nos llevó a realizar una técnica por abordaje único sub axilar, a través de la cual se diseca el músculo dorsal ancho y se transpone de forma anterior, obteniendo una sola incisión, la cual se mantiene oculta debajo del miembro superior adducido. Produciendo menor tiempo operatorio, menor tiempo de internación, disminuyendo el número de incisiones y logrando una adecuada fijación anterior del músculo dorsal ancho.

Palabras clave: reconstrucción mamaria, colgajo autólogo, síndrome de Poland

\section{ABSTRACT}

Poland's syndrome is a malformation associated with varying degrees of thoracic and upper limb abnormality, with an incidence of 1 in 30,000 live births.

This pathology generates the absence of the mammary gland and the major pectoral muscle as the most frequent malformation. Which produces an asymmetry at the chest level with loss of harmony which leads to both physical and psychological alteration at the level of the patient. For this reason, it is necessary to resolve this pathology with the least possible morbidity.

The proposal to generate the least morbidity led us to perform a single sub axillary approach technique, through which the latissimus dorsi muscle is dissected and transposed anteriorly, obtaining a single incision, which is kept hidden under the upper limb adduced. Producing less operative time, shorter hospitalization time, reducing the number of incisions and achieving an adequate anterior fixation of the latissimus dorsi muscle.

Keywords: breast reconstruction, autologous flap, Poland syndrome.

RESUMO

A sindrome de Poland é uma malformação associada a vários graus de anomalia torácica e de membros superiores, com uma incidência de 1 em 30.000 nascidos vivos.

Essa patologia gera a ausência da glândula mamária e do músculo pectoral principal como a malformação mais frequente. $O$ que produz uma assimetria ao nivel do peito com perda de harmonia que conduz a alterações fisicas e psicológicas ao nivel do paciente. Por isso, é necessário resolver essa patologia com a menor morbidade possivel

A proposta de gerar o mínimo de morbidade nos levou a realizar uma única técnica de abordagem subaxilar, por meio da qual o músculo grande dorsal é dissecado e transposto anteriormente, obtendo-se uma única incisão, que é mantida escondida sob o membro superior. aduzido. Produzindo menos tempo operatório, menor tempo de internação, reduzindo o número de incisões e conseguindo uma fixação anterior adequada do músculo grande dorsal.

Palavras-chave: reconstrução mamária, retalho autólogo, sindrome de Poland

\section{INTRODUCCIÓN}

El sindrome de Poland fue descrito por Alfred Poland en 1841. Constituye una malformación que asocia grados diversos de anomalias torácicas y del miembro superior homolateral, que resultan en la agenesia de fascículos esterno-costales del pectoral mayor ${ }^{(1)}$. Si bien se desconoce su etiología la hipótesis más aceptada es la interrupción del flujo sanguíneo en la arteria subclavia durante el desarrollo embrionario(3).

La incidencia de esta patología se da en aproximadamente 1 cada 30000 nacidos vivos, correspondiendo al $14 \%$ de todas las aplasias mamarias(2).

Su tratamiento consiste en la reconstrucción de las malformaciones torácicas, así como reconstrucción mamaria ya sea por abordaje convencional (más utilizado en nuestro medio) y abordaje laparoscópico. Para el abordaje convencional existen diferentes opciones tanto para las incisiones cutáneas, como para las técnicas de reconstrucción que comprenden implantación de prótesis pectorales y/o mamarias, relleno graso y/o rotaciones musculares y cutáneas ${ }^{14 .}$ 5.6)

La ausencia del pectoral mayor no solo resulta en la ausencia de simetría mamaria sino también en la ausencia del pilar anterior de la axila, elementos esenciales en la armonía torácica. La técnica de reconstrucción mamaria mediante la utilización del colgajo de dorsal ancho bipolar, en la cual se desinserta el tendón humeral del músculo dorsal ancho y lo 
reinserta a nivel del surco intertubercular del humero, recrea el pilar anterior de la axila.

Como se menciono previamente existen diferentes abordajes para la realización de esta técnica. Planteamos la utilización de un solo abordaje en cara lateral del tórax, subaxilar, disminuyendo la morbimortalidad y con menos $\operatorname{cicatrices}^{(7,8,9)}$

\section{CASO CLÍNICO}

Se trata de una paciente de 19 años con un síndrome de Poland con agenesia de la glándula mamaria derecha y músculos pectorales, sin malformaciones del miembro superior. Dos años antes se realizó la reconstrucción mamaria mediante colocación de un implante mamario a derecha, de silicona, anatómico, en un bolsillo subcutáneo, por abordaje periareolar.

Al momento de la consulta la paciente presentaba la cicatriz periareolar ensanchada, con un implante muy notorio y rotado, con una asimetría del surco submamario y una ausencia del pilar anterior de la axila, lo cual molestaba notoriamente a la paciente.

\section{(Figura 1)}

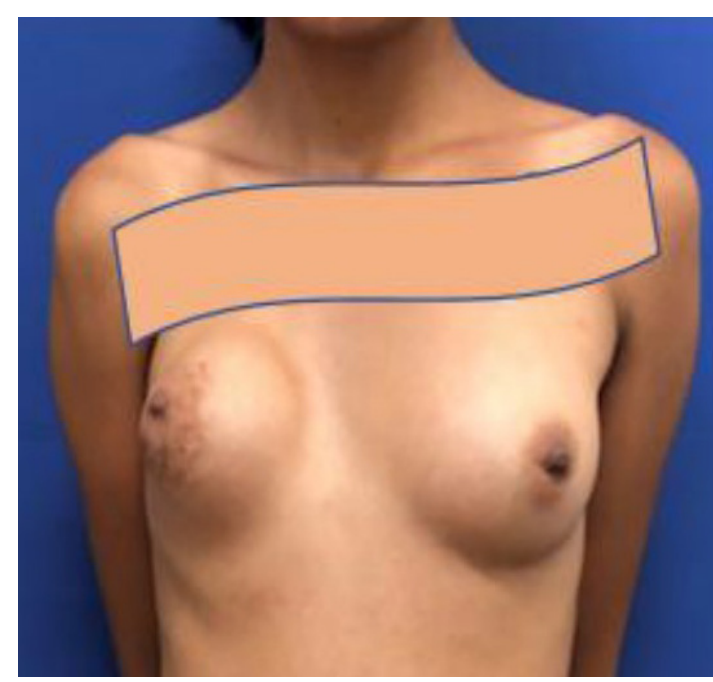

Figura 1. Paciente pre operatoria

Fuente: elaboración propia.

\section{TÉCNICA QUIRÚRGICA}

La marcación en la paciente es sentada o de pie, de forma que pueda reproducirse un adecuado surco sub mamario para planear la reconstrucción. Se marca la incisión en la línea medio-axilar de la cara lateral del tórax, vertical, comenzando en el hueco axilar y extendiéndose hacia caudal aproximadamente por 9-10 cm, hasta la sexta costilla. Se marca el surco submamario donde se va a fijar el músculo dorsal ancho. La posición del paciente en la mesa de operaciones es en decúbito lateral. Se realiza un abordaje único subaxilar. Se comienza disecando el musculo dorsal ancho, primero en su cara superficial y luego por su cara profunda cuidando de no lesionar el pedículo. Una vez se encuentra disecado se realiza la desinserción a nivel vertebral y lo más alejado posible a través del abordaje propuesto. Se aborda el bolsillo mamario a través del mismo abordaje, abordando la cápsula del implantey retirando el mismo. Se constata la rotación del implante, con la base ubicada lateralmente. Se realiza la capsulotomía circunferencial, separando su cara anterior de su cara posterior y reposicionado el surco submamario y el resto de los límites del bolsillo. A continuación, se realiza la liberación de la inserción humeral del dorsal ancho, transponiendo el músculo a forma de colgajo en isla hacia el nuevo bolsillo mamario, cuidando no rotar el pediculo. (Figura 2). Se realiza luego la reinserción del tendón del dorsal ancho al surco intertubercular del humero, en la cara anterior del cuello del húmero con puntos al periostio, con ethibond 2.0 (Ethibond, Ethicon, J\&J), por delante del paquete axilar. Esta reinserción tendinosa permite recrear el pilar anterior de la axila y el cuerpo muscular formará la cara anterior el nuevo bolsillo mamario, ubicándose por detrás del plano cutáneo incluyendo la hoja anterior de la cápsula periprotésica. Se fijará el músculo en los límites del bolsillo mamario mediante puntos percutáneos. Se coloca el implante por detrás del colgajo muscular, redondo, texturizado de 250cc. Cierre del abordaje. Por último, realizamos la corrección de la areola, mediante resección de la cicatriz periareolar, sin comunicar la herida con el bolsillo mamario. Se deja drenaje aspirativo en el dorso que se mantuvo por 48 horas cuando el gasto fue escaso.

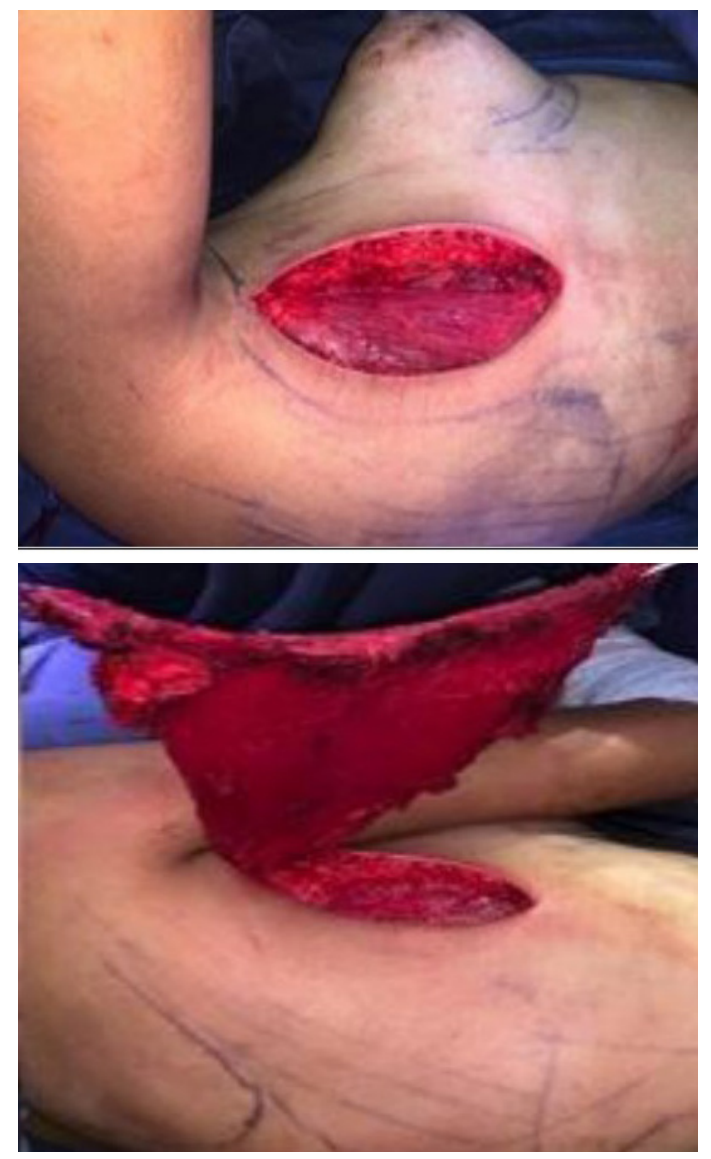

Figura 2. Abordaje con dorsal ancho disecado y rotado

Fuente: elaboración propia. 


\section{RESULTADOS}

La recuperación postoperatoria fue muy buena, sin complicaciones, pudiendo otorgarse el alta a las 72 horas de la cirugia. Se evaluaron los resultados a los 2 meses y al año. La reconstrucción mamaria realizada con colgajo muscular de dorsal ancho bipolar permitió lograr un contorno mamario muy satisfactorio, armónico con el contralateral, aunque con un volumen mayor, que requerirá una simetrización posterior, mediante un aumento mamario a izquierda.

Se logró una aceptable recreación del pilar anterior de la axila, rellenándose la depresión que presentaba a ese nivel. La corrección de la cicatriz periareolar permitió lograr una areola más simétrica con la contralateral, con menos secuelas. (Figura 3).

El abordaje subaxilar permitió realizar el procedimiento dejando una sola cicatriz, algo pigmentada, relacionada con el tinte racial de la paciente.
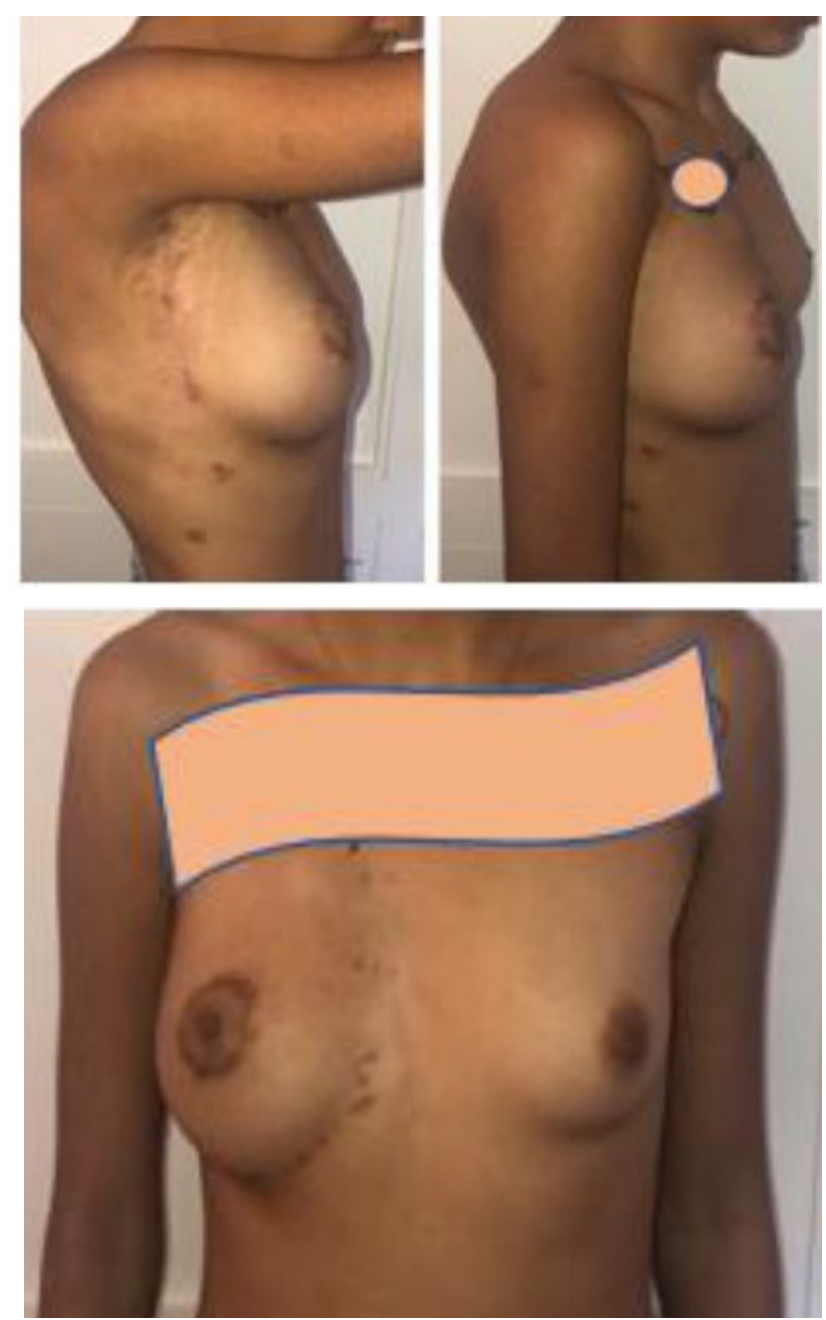

Figura 3. Post operatorio.

Fuente: elaboración propia.

Los puntos transcutáneos de fijación del músculo dorsal ancho dejaron la presencia de pequeñas cicatrices puntiformes hipertróficas, probablemente también relacionadas con el color de piel de la paciente, en tratamiento actual mediante inyección de corticoides.

\section{DISCUSIÓN}

La transferencia bipolar del dorsal ancho para la reconstrucción torácica y mamaria en el Sindrome de Poland fue descrita por Buchanan en 2016. Esta técnica permite corregir los dos polos fundamentales para devolver la apariencia anatómica natural del tórax y su simetria. Por un lado, recrear el pilar anterior de la axila, y por otro, contribuir a corregir la región pectoral en el hombre, o la región mamaria en la mujer.

En 2016, Wafta et al.(7) plantea la realización de esta técnica por un abordaje vertical único medio-axilar, en la cara lateral del tórax. Este abordaje permite un acceso completo al musculo dorsal ancho en el dorso, a su pedículo y a su tendón de inserción humeral en la axila, permitiendo la desinserción y reinserción de este. Por este mismo abordaje se accede a la mama, se puede disecar el bolsillo para la colocación del implante y del cuerpo muscular del dorsal ancho, y prácticamente toda la reconstrucción puede realizarse en esta posición.

Si bien la técnica laparoscopia presenta menor cicatriz cutánea, en la paciente no permite realizar la disección y reconstrucción de forma bipolar del músculo dorsal ancho así como también requiere de formación especial en técnicas laparoscópicas lo cual incrementa la curva de aprendizaje.

Por lo antes expresado la elección de nuestra técnica quirúrgica nos aporta un menor tiempo quirúrgico ya que se evita la rotación de la paciente en el intraoperatorio, con una recuperación también más rápida acortando los dias de internación. Nos permite acceder a las dos inserciones del músculo para recrear el pilar anterior de la axila, evitando la cicatriz en dorso, y cicatrices complementarias en la mama, fundamental en una paciente sin cicatrices mamarias previas, como generalmente ocurre en el sindrome de Poland, a diferencia de la reconstrucción mamaria secundaria a una mastectomía. Permitiendo realizar esta técnica a cirujanos jóvenes, sin requerir formación adicional en laparoscopia lo que alargaría la curva de aprendizaje de la técnica.

La paciente deberá someterse a cirugía de simetrización contralateral para lograr la armonía torácica, lo cual se evito realizar en el mismo tiempo quirúrgico a la espera de una correcta cicatrización y eventual atrofia muscular cambiando el aspecto final de volumen mamario.

\section{CONCLUSIÓN}

La reconstrucción mamaria con colgajo muscular de dorsal ancho bipolar por abordaje único subaxilar vertical asociada a un implante mamario es una técnica segura y reproducible, lográndose resultados 
cosméticos y funcionalmente muy buenos.

Permite realizar la reconstrucción de la mama y del pilar anterior de la axila mediante el uso de un solo músculo.

El abordaje subaxilar vertical permite realizar toda la cirugía sin cambios de decúbito, lo cual acorta los tiempos quirúrgicos. La cicatriz resultante queda oculta por el brazo de la paciente, evitando cicatrices en dorso y en la mama.

\section{REFERENCIAS}

1. Chichery, A. \& Jalbert, F. \& Foucras, Lionel \& Grolleau, J.-L \& Chavoin, Jean-Pierre. (2006). Sindrome de Poland. EMC - Cirugia Plástica Reparadora y Estética. 14. 1-17. 10.1016/ S1634-2143(06)47927-9

2. McGillivray BC, Lowry RB. Poland syndrome in British Columbia: incidence and reproductive experience of affected persons. Am J Med Genet. 1977:1:65-74.

3. Mingulla-Sola J. Poland's syndrome: a review of 38 cases. Am Esp Pedriatr. 1998;48:143-147.

4. Baratte A, BodinF, DelPin D, et al. [Poland's syndrome in women: therapeutic indications according to the grade. Apropos of 11 cas- es and review of the literature]. Ann Chir Plast Esthet. 2011:56:33-42.

5. Dos Santos Costa S, Blotta RM, Mariano MB, Meurer L, Edelweiss MI. Aesthetic improvements in Poland's syndrome treatment with omentum flap. Aesthetic Plast Surg. 2010;34:634-639.

6. Pinsolle V, Chichery A, Grolleau JL, Chavoin JP. Autologous fat injection in Poland's syndrome. J Plast Reconstr Aesthet Surg. 2008:61:784-791.

7. Watfa W, di Summa PG, Raffoul W. Bipolar Latissimus Dorsi Transfer through a Single Incision: First Key-Step in Poland Syndrome Chest Deformity. Plast Reconstr Surg Glob Open. 2016;4(8):e847. doi: 10.1097/GOX.0000000000000851. PMID: 27622115: PMCID: PMC5010338.

8. Arslan E, Unal S, Demirkan F, et al. Poland's syndrome with rare deformities: reconstruction with latissimus dorsi muscle through a single short incision. Scand J Plast Reconstr SurgHand Surg. 2003:37:304-306.

9. Buchanan P, Leyngold M, Mast BA. Bipolar latissimus dorsi transfer for restoration of pectoralis major function in poland syn- drome. Ann Plast Surg. 2016;77:85-89.

\section{Nota de contribución:}

En cuanto al aporte del trabajo de los autores se realizo la concepcion y diseño del estudio por parte de Maximiliano Juri, la recolceccion de datos o realización de los experimentos se realizo en conjunto con Maximiliano Juri y Virginia Giachero, el analisis e interpretación de los datos o resultados por parte de Maximiliano Juri y Virginia Giachero, la elabroacion de uin borrador de manuscrito realizada por Maximiliano Juri, escritura del manuscrito por parte de Maximiliano Juri, se superviso el trabajp por parte Virginia Giachero y la colección y manejo de las muestras provenientes del paciente fue tomada por Maximiliano Juri y Virginia Giachero.

\section{Nota del Editor:}

El presente manuscrito fue aprobado para su publicación por el Dr. Crestanello.

Recibido: 22/12/2020

Aceptado: 20/04/2021 